\title{
Conditioned cardiac acceleration under spreading cortical depression ${ }^{1}$
}

\author{
LINDA BURGOYNE ${ }^{2}$, ROBERT POTE AND NELSON FREEDMAN \\ QUEEN'S UNIVERSITY, KINGSTON, ONTARIO
}

An accelerated cardiac response was conditioned on two successive days under four spreading depression (SD) conditions; saline control (NSD), bilaterally depressed (BSD), same hemisphere depressed unilaterally (USD), and alternate hemispheres depressed unilaterally (ASD) on both days. All four groups were as readily conditioned to this subcortically mediated autonomic response.

Recent studies have clearly demonstrated that motor responding in rats is severely depressed under BSD (Freedman \& Lash, 1966; Moelis, 1964; Tapp, 1962; Winocur, 1965). Presumably, any learning study involving a motor response under SD is confounded by this motor impairment. However, Mogenson \& Peterson (1966) have shown that a well trained cardiac acceleration conditioned prior to $10 \% \mathrm{KCl}$ induced SD is unaffected by later depression.

In contrast to motor responding, and providing SD is principally a cortical phenomenon, autonomic or subcortically elaborated responses should not only be unaffected by subsequent $\mathrm{KCl}$ application, but also should be completely conditionable under KCl. Furthermore, such responses should show complete transfer from a trained hemisphere to an untrained one. The present study investigated these hypotheses using heart rate acceleration responses which are unconfounded by motor impairment.

Method

Thirty-two male albino rats were anesthetized with Nembutal (.01 cc/100 g body weight) and .15 cc atropine sulphate. The Ss were then surgically prepared for SD according to the chronic procedure described by Tapp (1962). Heart rate electrodes composed of stainless steel wire were looped through the dorsal skin just posterior to the forepaws.

After 2 to $2-1 / 2$ days of recovery, the Ss were conditioned in an $18 \times 18 \times 24$ in. deep box containing a grid floor and loudspeaker. The CS was $10 \mathrm{sec}$ increase in the intensity of the background white noise (65-85 dB); the UCS was a 1 sec, $1.6 \mathrm{~mA}$ scrambled foot shock, and the variable intertrial interval (ITI) averaged $4 \mathrm{~min}$. The EKG was recorded on a Grass Model 5 polygraph $10 \mathrm{sec}$ before the CS, $10 \mathrm{sec}$ during the CS, and $10 \mathrm{sec}$ after the shock. The polygraph was automatically turned off during shock administration.

Four groups of Ss were run in this apparatus over a 2 day period, separated by 2 to $2-1 / 2$ days. A saline control group (NSD) received sterile Ringer's solution in their tubes leading to the dural membrane on both days; a bilateral depressed group (BSD), $25 \% \mathrm{KCl}$ on both days; a unilateral depressed group (USD), 25\% $\mathrm{KCl}$ same hemisphere each day; and an alternating depressed group (ASD), $25 \% \mathrm{KCl}$ on first one hemisphere, then the other. On the first day, the appropriate solution was applied and the EKG was recorded for 10 trials, no $\mathrm{CS}$, to allow adaptation to the situation. Adaptation was followed by 10 trials of CS alone, to adapt EKG to this novel stimulus. Finally, S was given 25 CS-UCS pairings. On Day 2, $S$ was depressed and EKG recorded during 25 further CS-UCS pairings.

To determine whether results of these particular procedures could be attributed to pseudoconditioning, two additional unoperated control groups were run. One group received the same adaptation, tone alone, and CS-UCS pairings as the experimental Ss on the two days. The other group received adaptation and tone alone, but $25 \mathrm{CS}$ and 25 UCS trials unpaired. Results and Discussion

During adaptation to the experimental situation, there was a significant decline $(F=10.80, d f=9 / 276$, $p<.001$ ) in heart rate (HR) from 492 to 435 beats/ min for all four groups. On the CS adaptation trials the four treatment groups were also the same on both pre-CS HR (10 sec before CS onset) and during-CS HR (during $10 \mathrm{sec}$ of $\mathrm{CS}$ ), though the during-CS HR was significantly higher $(\mathrm{F}=4.69, \mathrm{df}=1 / 556, \mathrm{p}<.05)$ by about 6 beats/min for all groups. It was concluded that the presence or absence of SD, whether BSD, USD, or ASD, does not differentially affect HR adaptation either to the experimental situation or the CS.

The trend analysis on the CS-UCS pairings on the two days shows no overall group differences. The HR difference between the before-CS and during-CS measures was at this point 16 beats $/ \mathrm{min}(F=186.48$, df $=1 / 2772, p<.001$ ). The measures by trials by days interaction (Fig. 1) was significant ( $F=2.11, \mathrm{df}=24$ / $2772, p<.005)$ indicating the $H R$ was better conditioned on Day 2, though the treatment groups were not different in this regard. It is probable that the large adaptation effect due to the introduction of the UCS on Day 1 interfered with the learning process on that day. That the Ss were learning on Day 1, however, is shown by the large divergence between the two HR measures appearing as early as Trial 3, on Day 2, for all groups. As with situational and CS adaptation, the BSD, ASD, USD, and NSD groups did not differ in conditionability over the two days of training.

The unoperated CS-UCS paired groups showed significant $H R$ acceleration $(F=4.03$, df $=24 / 336, p$ 

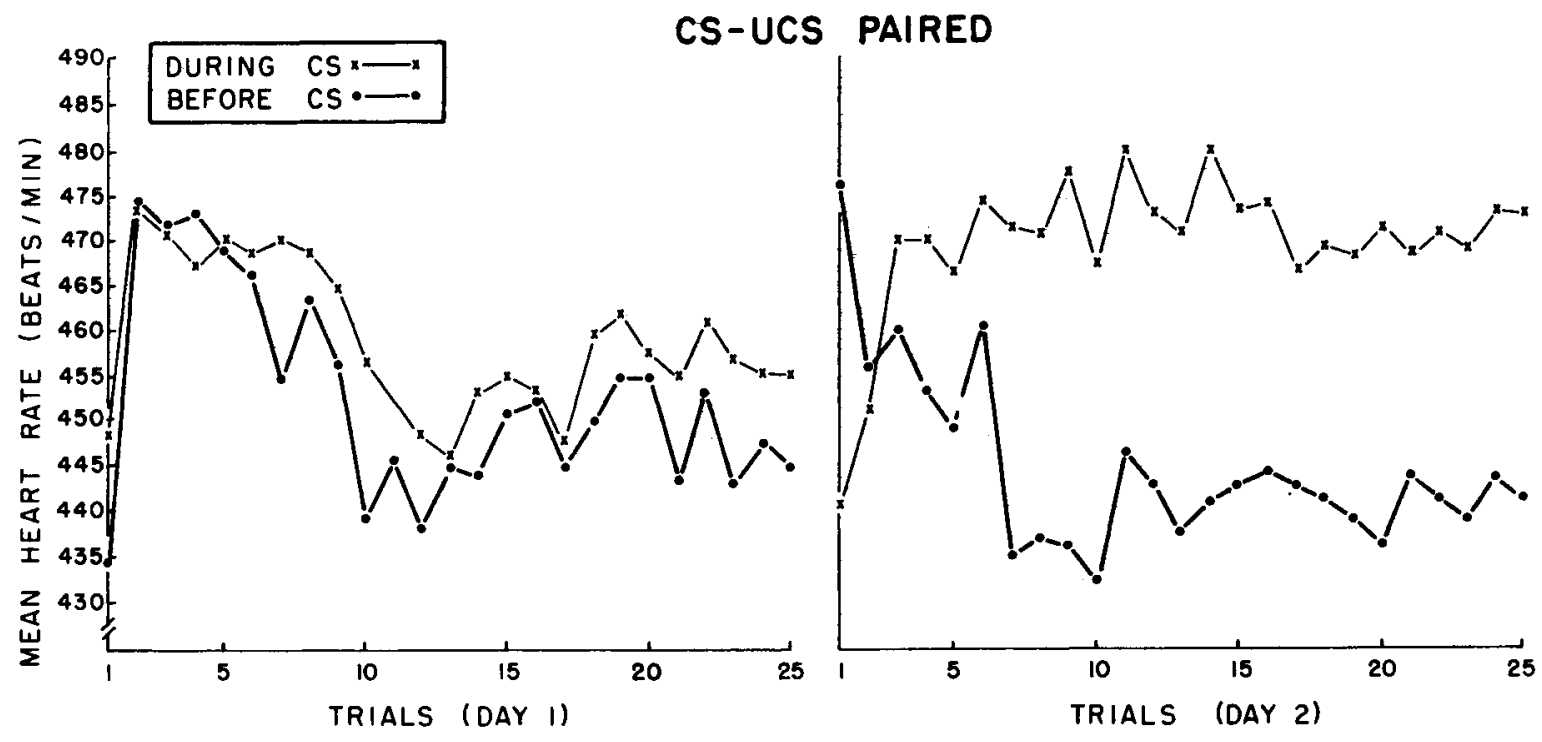

Fig. 1. Before- and during-CS HR averaged over treatment groups on the two training days. The conditioning effect shown by the difference between these measures is larger on Day 2 .

$<.001)$ over the two days and were more similar to the operated groups than the unpaired group. The unpaired group showed no significant differences in pre- and during-CS measures over trials. Thus, the performance of the operated Ss was neither impaired by SD, nor could it be attributable to a pseudoconditioning process.

These data demonstrate that Ss readily learn an accelerated HR response irrespective of the chemical disruption of cortical electrical activity. Unlike conditioned somatic responses, the BSD group performed as well as normal controls, sham operated or not. The ASD group who came to the learning situation on Day 2 with only "naive" cortex also responded as well as normals. Since SD primarily affects cortical functioning (Ochs, 1962), these results provide tentative support for the hypothesis that cardiac conditioning does not depend on functional cortical tissue. Furthermore, as might be expected of subcortical activity, this conditioning showed positive subcortical transfer from training with one hemisphere depressed and testing contralaterally.

\section{References}

FREEDMAN, N., \& LASH, L. Conditioned avoidance decrement under spreading depression. Psychon Sci, 1966, 5, 411-412.

MOELIS, I. An evaluation of the effects of spreading cortical depression on motor aspects of performance. (Doctoral dissertation, University of Illinois) Ann Arbor, Mich: University Microfilms, 1963. No. 646115 .

MOGENSON, G. J., \& PETERSON, R. Effect of spreading cortical depression on cardiac and somatomotor conditioned responses. Canad. J. Physiol. Pharmacol, 1966, 44, 39-45.

OCHS, $S$. The nature of spreading depression in neural networks. Int. Rev. Neurobiol, 1962, 5, 1-67.

TAPP, J. T. Reversible cortical depression and avoidance behavior in the rat. J. comp. physiol. Psychol., 1962, 55, 306-308.

WINOCUR, G. Bilateral spreading depression and avoidance learning in the rat. Psychon. Sci, 1965, 3, 107-108.

\section{Notes}

1. Based on an Honors Thesis by L. B. presented in part at Canadian Psychological Association Meetings, May, 1967. Supported by McGlaughlin Science, National Research Council, and Department of University Affairs Grants to N. F. We thank E. Griffiths and B. Osborne for technical assistance.

2. Presently at McGill University. Reprints may be obtained from L. B. or N. F. 